\title{
Coupling Analysis for Systolic, Diastolic and RR Interval Time Series Using Multivariable Fuzzy Measure Entropy
}

\author{
Lina Zhao ${ }^{1}$, Shoushui $\mathrm{Wei}^{1}$, Hong Tang ${ }^{2}$, Chengyu $\mathrm{Liu}^{3}$ \\ ${ }^{1}$ School of Control Science and Engineering, Shandong University, Jinan, China \\ ${ }^{2}$ Department of Biomedical Engineering, Dalian University of Technology, Dalian, China \\ ${ }^{3}$ School of Instrument Science and Engineering, Southeast University, Nanjing, China
}

\begin{abstract}
In this study, we performed a new multivariate fuzzy measure entropy (mvFME) analysis on the recorded multivariate systolic, diastolic and RR interval time series. Twenty healthy young male subjects $(24.2 \pm 1.9$ years $)$ were enrolled. For each subject, both ECG and aortic phonocardiogram (PCG) signals were simultaneously recorded for 5 minutes, under two physiological states respectively: rest and after stair climbing. RR interval time series were constructed from locating $Q R S$ complexes in ECG signal by Pan \& Tompkins method. Systolic and diastolic time series were constructed from identifying the beginning of the first and second sound in PCG signal by Springer's hidden semi-Markov model segmentation method. The results showed that, compared with rest state, after stair climbing state has significant lower mvFME values for both univariate and multivariate time series analysis (all $P<0.01$, except univariate systolic time series with $P<0.05)$. The mean $m v F M E$ values decreased from using univariate to multivariate time series for both rest and after stair climbing states. This study shows physical activity changes the coupling relationship in cardiac interval time series. Meanwhile, coupling between $R R$ and systolic time series reports larger mvFME values than coupling between $R R$ and diastolic time series.
\end{abstract}

\section{Introduction}

Quantitative analysis of the variability of RR interval time series, i.e., heart rate variability (HRV) analysis, provides an estimate of autonomic nerve control of the heart and is an important tool for evaluating the function of cardiovascular (CV) system [1]. RR interval consists of systolic time interval (STI) and diastolic time interval (DTI), whose time series also present the variability characteristics. Liu et al. studied the univariate HRV, STI variability (STIV) and DTI variability (DTIV), and reported that compared with STIV, DTIV is more obvious to follow the change of HRV [2].

The complex dynamical behavior of $\mathrm{CV}$ system is not only reflected within the single time series, but more importantly to be reflected across multiple data channels. The coupling relationship between RR and STI time series were analyzed using a multivariate sample entropy (mvSE) method [3-5]. In 2016, Zhao et al. proposed a new multivariate fuzzy measure entropy (mvFME) analysis for analyzing multivariate RR interval, first (S1) and second (S2) heart sound amplitude time series [6], and the new mvFME method showed better statistical stability and discrimination ability for multivariate time series analysis than the traditional mvSE method.

In this study, we performed the new mvFME analysis on multivariate RR, STI and DTI time series, to compare the mvFME differences between two physiological states: rest state and after stair climbing state.

\section{Methods}

\subsection{Subjects}

Twenty healthy young male subjects $(24.2 \pm 1.9$ years $)$ were enrolled in this study. All subjects gave their written informed consent, and confirmed that they had not participated in any other 'clinical trial' within the previous three months. The study obtained a full approval from the Clinical Ethics Committee of the Second Hospital of Dalian Medical University and all clinical investigation was conducted according to the principles of expressed in the Declaration of Helsinki. Table 1 depicts the details for the involved subjects.

Table 1. Demographic data for the subjects studied.

\begin{tabular}{lc}
\hline Variables & Values \\
\hline Age (year) & $24.2 \pm 1.9$ \\
Height $(\mathrm{cm})$ & $174 \pm 4$ \\
Weight $(\mathrm{kg})$ & $64 \pm 7$ \\
HR (beats/min) & $69 \pm 9$ \\
SBP (mmHg) & $121 \pm 9$ \\
DBP (mmHg) & $65 \pm 7$ \\
\hline
\end{tabular}

Note: data are expressed as mean \pm standard deviation (SD). HR: heart rate, SBP: systolic blood pressure, DBP: diastolic blood pressure. 


\subsection{Experimental procedure}

For each subject, standard limb lead-I ECG and phonocardiogram (PCG) signals were simultaneously recorded with a sample rate of $2,000 \mathrm{~Hz}$. PCG signal was recorded by the microphone sensors (MLT201, AD instrument, Australia) at the aortic auscultation positions [7; 8]. Signals were firstly recorded for about 5 minutes at rest state. Then the subject was asked to do the activity of stair climbing for 120 stairs. After the stair climbing, the subject was asked immediately to start the signal recording for another 5 minutes. Before signal recording, each subject had a rest for 10 minutes. Heart rate (HR) and blood pressure (BP) values, including systolic blood pressure (SBP) and diastolic blood pressure (DBP), were measured by an automatic electronic sphygmomanometer (HEM-7200, OMRON, Japan).
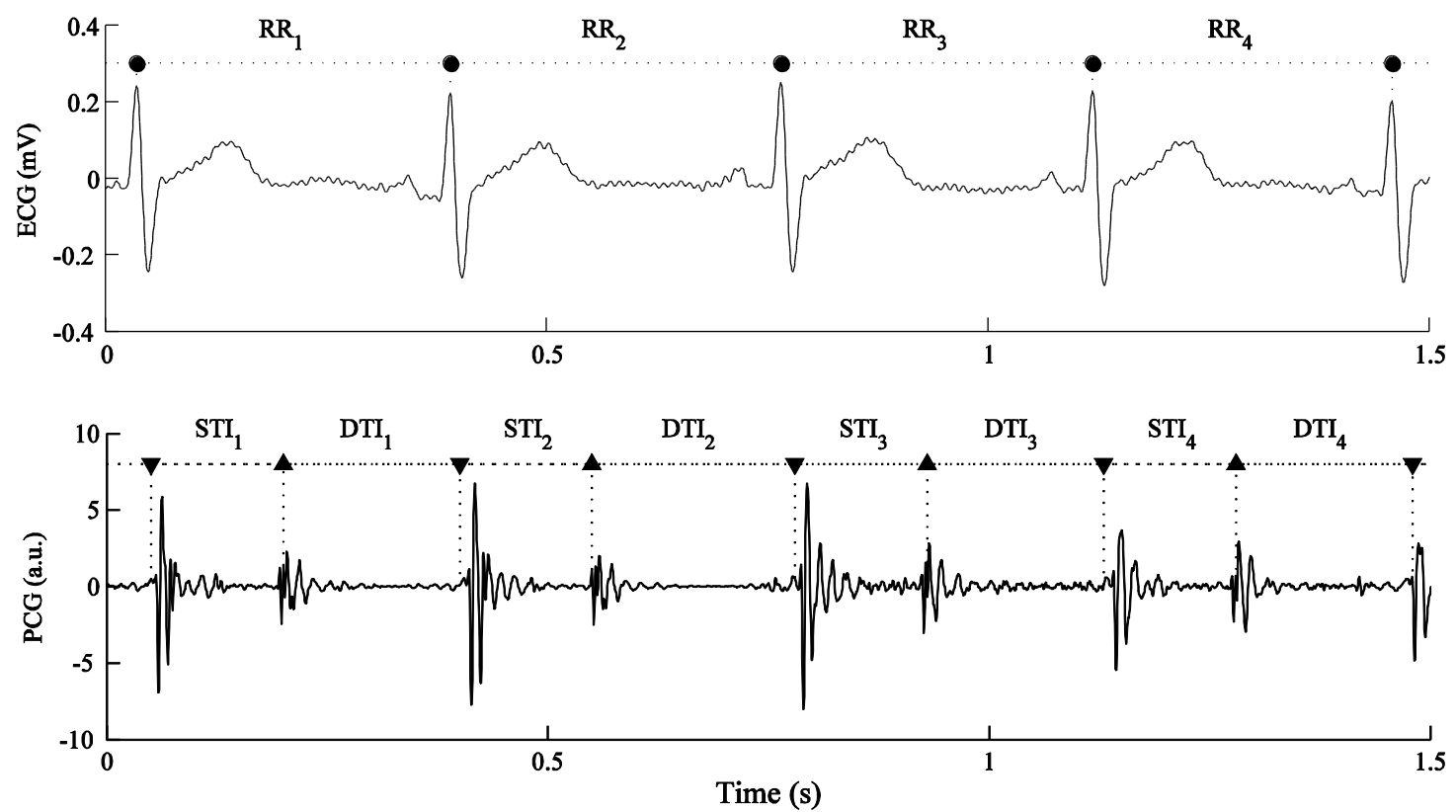

Figure 1. Simultaneously recorded ECG and phonocardiogram (PCG) signals. The detected R-wave peaks are denoted as "•", the onset of the first and second heart sounds are denoted as " $\boldsymbol{\nabla}$ " and " $\mathbf{\Delta}$ " respectively.

(A1)

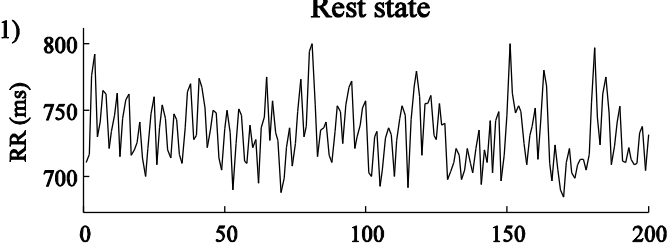

(B1)

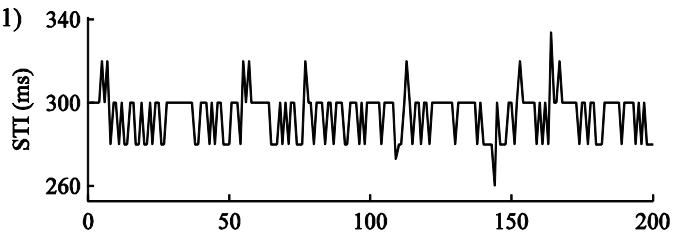

(C1)

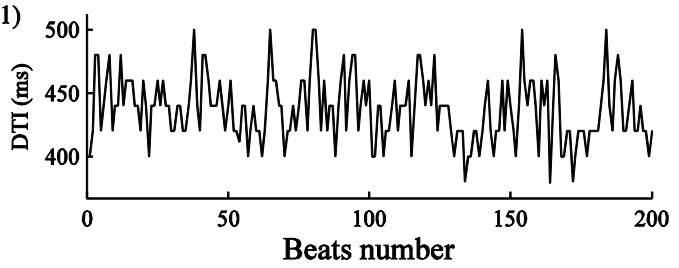

(A2)

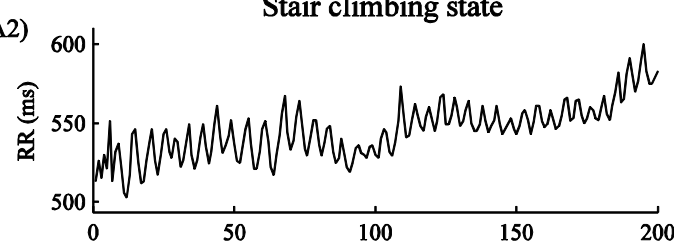

(B2)

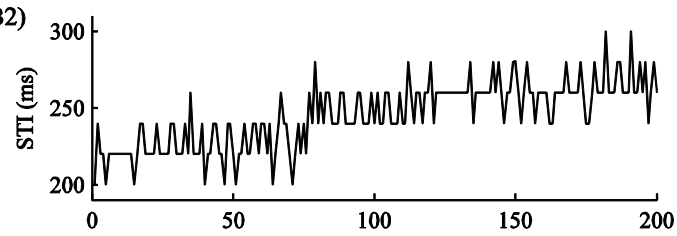

(C2)

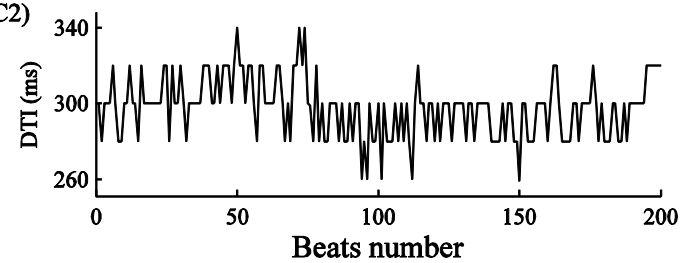

Figure 2. Example of RR, STI and DTI series from a subject during the two states for 200 beats. 


\subsection{Signal processing}

ECG signal was firstly filtered using a $0.05-40 \mathrm{~Hz}$ band-pass filter. Pan \& Tompkins method [9] was used to locate the $\mathrm{R}$ peaks and thus the RR interval time series were constructed. RR intervals with ectopic beats were detected and excluded using the combination method [10]. PCG signals were firstly filtered using a $20-200 \mathrm{~Hz}$ bandpass filter. Then Springer's hidden semi-Markov model (HSMM) segmentation method [11] was used to segment each PCG signal to detect the onsets for S1 and S2 heart sounds. Figure 1 shows waveform examples, and the corresponding R peak location, the onsets of the first (S1) and second (S2) heart sounds. Finally, RR, STI (the interval between the onsets of S1 and S2 in the current heart circle) and DTI (the interval between the onset of S2 in the current heart circle and the onset of S1 in the next heart circle) were constructed. Figure 2 shows the examples from both rest and after stair climbing states. For each time series, only the first 200 beats were shown.

\subsection{Multivariate fuzzy measure entropy}

mvFME method was recently developed from the fuzzy measure entropy (FuzzyMEn) by Liu et al [12; 13]. The details of the calculation process can refer to [6]. We did not detail the calculation process here. The parameter setting for mvFME used the suggested values in our previous studies $[14 ; 15]$. mvFME is the same as the FuzzyMEn for univariate time series analysis. In this study, the CV time series used for mvFME analysis are summarized as:

- Univariate: RR, STI and DTI;

- Bivariate: RR \& STI, RR \& DTI and STI \& DTI;

- Trivariate: RR \& STI \& DTI.

\subsection{Statistical analysis}

The overall means and SDs of mvFME were obtained separately for the two physiological states, i.e., rest state and after stair climbing state. The differences between the two states were compared using a student's $t$-test (SPSS 19.0 software package). A value of $P<0.05$ was considered statistically significant.

\section{Results}

Figure 3 and Table 2 give the mvFME results from the univariate, bivariate and trivariate time series for both rest and after stair climbing states. All mvFME values in the after stair climbing state were significant lower than those in the rest state (all $P<0.01$, except for the univariate STI series $P<0.05$ ). The compared results of mvFME in rest and after stair climbing states were: $2.17 \pm 0.34$ vs. $0.93 \pm 0.37$ for RR, $1.83 \pm 0.28$ vs. $1.51 \pm 0.50$ for systolic, $2.05 \pm 0.48$ vs. $1.32 \pm 0.52$ for diastolic, $1.71 \pm 0.24$ vs. $0.76 \pm 0.22$ for RR \& systolic coupling, $1.53 \pm 0.33$ vs.
$0.71 \pm 0.28$ for $\mathrm{RR} \&$ diastolic coupling, $1.63 \pm 0.29$ vs. $0.92 \pm 0.30$ for systolic \& diastolic coupling, and $1.22 \pm 0.23$ vs. $0.60 \pm 0.19$ for all three time series coupling.

For both rest and stair climbing states the mean mvFME values decreased from using univariate to multivariate time series (Figure 3 and Table 2).

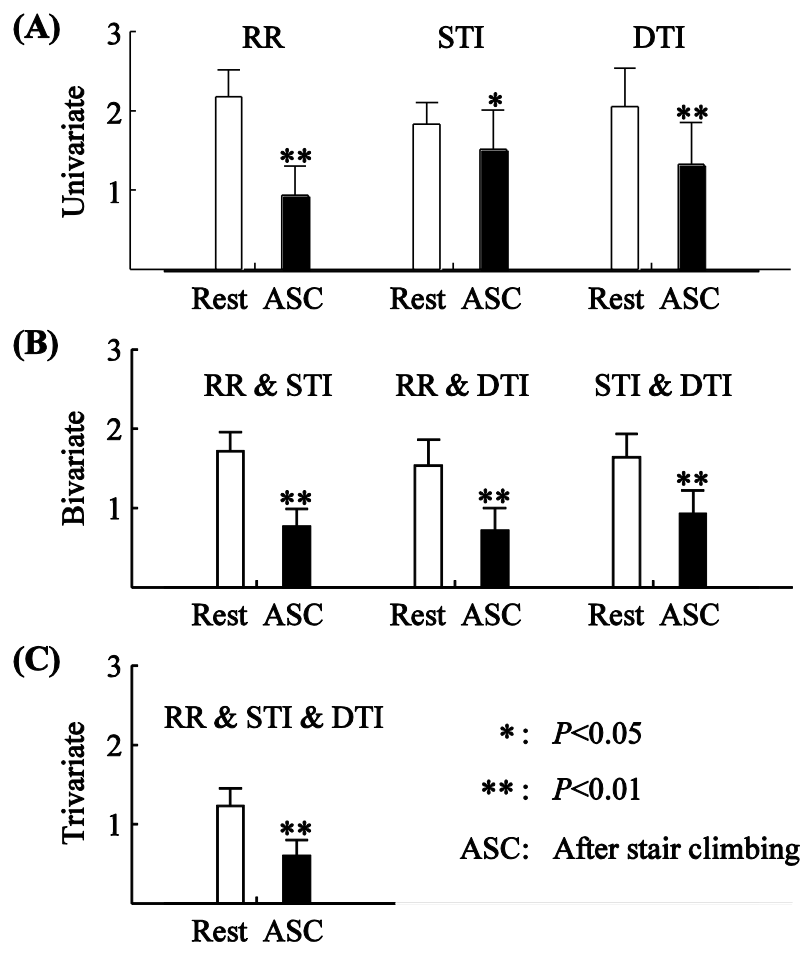

Figure 3. Statistical results of the univariate mvFME (A), bivariate mvFME (B) and trivariate mvFME (C) for the two physiological states.

Table 2. Results (mean $\pm \mathrm{SD}$ ) and statistical $P$-values of the univariate and multivariate mvFME for the rest and after stair climbing (ASC) states.

\begin{tabular}{cccc}
\hline Time series & Rest & ASC & $P$-values \\
\hline Univariate & & & \\
RR & $2.17 \pm 0.34$ & $0.93 \pm 0.37$ & $<0.01$ \\
STI & $1.83 \pm 0.28$ & $1.51 \pm 0.50$ & $<0.05$ \\
DTI & $2.05 \pm 0.48$ & $1.32 \pm 0.52$ & $<0.01$ \\
Bivariate & & & \\
RR \& STI & $1.71 \pm 0.24$ & $0.76 \pm 0.22$ & $<0.01$ \\
RR \& DTI & $1.53 \pm 0.33$ & $0.71 \pm 0.28$ & $<0.01$ \\
STI \& DTI & $1.63 \pm 0.29$ & $0.92 \pm 0.30$ & $<0.01$ \\
Trivariate & & & \\
RR \& STI \& DTI & $1.22 \pm 0.23$ & $0.60 \pm 0.19$ & $<0.01$ \\
\hline
\end{tabular}

\section{Discussions}

In clinical practice, STI and DTI can be easily acquired by simultaneously recording ECG and PCG signals. PCG signal processing has been a concern since the success of the PhysioNet/Computing in Cardiology Challenge 2016 
$[16 ; 17]$. This study analyzed the coupling characteristics for RR, STI and DTI time series under two physiological states. The decrease of the univariate mvFME in the stair climbing state confirms the significant complexity loss in RR, STI and DTI time series. It is worth to note that the decrease of complexity in HRV is mainly from the decrease in DTI time series.

The bivariate and trivariate mvFME values in the stair climbing state were also significantly lower than those in the rest state, indicating that the decrease of the cross-time series coupled dynamics. When using the multivariate (bivariate or trivariate) mvFME, the statistical significances between the two physiological states increased, confirming that the multivariate analysis could give a better understanding of the underlying CV system dynamics.

\section{Conclusion}

This study used the recently introduced mvFME method to analysis both the univariate and multivariate $\mathrm{CV}$ time series variability and to compare the differences between the rest and stair climbing states. The results indicate that physical activity changes the coupling relationship in cardiac interval time series. Meanwhile, coupling between RR and systolic time series reports larger mvFME values than coupling between $R R$ and diastolic time series.

\section{Acknowledgement}

This work was supported by the National Natural Science Foundation of China (61671275, 61471081 and 61201049), and the project of Southeast-Lenovo wearable Heart-Sleep-Emotion intelligent monitoring Lab.

\section{References}

[1] Task Force of the European Society of Cardiology and the North American Society of Pacing and Electrophysiology. Heart rate variability: standards of measurement, physiological interpretation and clinical use. Circulation. 1996; 93: 1043-1065.

[2] Liu C Y, Liu C C, Li L P, Zhang Q G, Li B. Systolic and diastolic time interval variability analysis and their relations with heart rate variability. 3rd International Conference on Bioinformatics and Biomedical Engineering. 2009; 1-4.

[3] Liu C Y, Zheng D C, Zhao L N, Li P, Liu C C, Murray A. Analysis of cardiovascular time series using multivariate sample entropy: A comparison between normal and congestive heart failure subjects. Computing in Cardiology. 2014; 41: 237-240.

[4] Ahmed M U, Mandic D P. Multivariate multiscale entropy analysis. IEEE Signal Proc Let. 2012; 19: 91-94.

[5] Ahmed M U, Mandic D P. Multivariate multiscale entropy: a tool for complexity analysis of multichannel data. Physical Review E. 2011; 84: 061918.

[6] Zhao L N, Wei S S, Tang H, Liu C Y. Multivariable fuzzy measure entropy analysis for heart rate variability and heart sound amplitude variability. Entropy. 2016; 18: 430.

[7] Tang H, Li T, Qiu T S, Park Y. Segmentation of heart sounds based on dynamic clustering. Biomed Signal Process Control. 2012; 7: 509-516.

[8] Tang H, Li T, Qiu T S. Noise and disturbance reduction for heart sounds in the cycle frequency domain based on non-linear time scaling. IEEE T Biomed Eng. 2010; 57: 325-333.

[9] Pan J, Tompkins W J. A real-time QRS detection algorithm. IEEE T Biomed Eng. 1985; 32: 230-236.

[10] Liu C Y, Li L P, Zhao L N, Zheng D C, Li P, Liu C C. A combination method of improved impulse rejection filter and template matching for identification of anomalous intervals in electrocardiographic RR sequences. J Med Biol Eng. 2012; 32: 245-250.

[11] Springer D B, Tarassenko L, Clifford G D. Logistic regression-HSMM-based heart sound segmentation. IEEE Trans Biomed Eng. 2015; In press:

[12] Liu C Y, Li K, Zhao L N, Liu F, Zheng D C, Liu C C, Liu $\mathrm{S} \mathrm{T}$. Analysis of heart rate variability using fuzzy measure entropy. Comput Biol Med. 2013; 43: 100-108.

[13] Liu C Y, Zhao L N. Using Fuzzy Measure Entropy to improve the stability of traditional entropy measures. Computing in Cardiology. 2011; 31: 681 - 684.

[14] Zhao L N, Wei S S, Zhang C Q, Zhang Y T, Jiang X E, Liu F, Liu C Y. Determination of sample entropy and fuzzy measure entropy parameters for distinguishing congestive heart failure from normal sinus rhythm subjects. Entropy. 2015; 19: 6270-6288.

[15] Liu C Y, Zhang C Q, Zhang L, Zhao L N, C. L C, Wang $\mathrm{H} \mathrm{J}$. Measuring synchronization in coupled simulation and coupled cardiovascular time series: A comparison of different cross entropy measures. Biomed Signal Process Control. 2015; 21: 49-57.

[16] Liu C Y, Springer D B, Li Q, Moody B, Juan R A, Chorro F J, Castells F, Riog J M, Silva I, Johnson A E W, Syed Z, Schmidt S E, Panadaniil C D, Hadjileontiadis L, Naseri H, Moukadem A, Dieterlen A, Brandt C, Tang H, Samieinasab M, Samieinasab M R, Sameni R, Mark R G, Clifford G D. An open access database for the evaluation of heart sound algorithms. Physiol Meas. 2016; 37: 21812213.

[17] Clifford G D, Liu C Y, Moody B, Springer D, Silva I, Li Q, Mark R G. Classification of normal/abnormal heart sound recordings: the PhysioNet/Computing in Cardiology Challenge 2016. Computing in Cardiology. 2016; 43: 609-612.

Address for correspondence.

Shoushui Wei (sswei@sdu.edu.cn)

School of Control Science and Engineering

Shandong University, Jinan, China

or

Chengyu Liu (bestlcy@sdu.edu.cn)

School of Instrument Science and Engineering

Southeast University, Nanjing, China 